
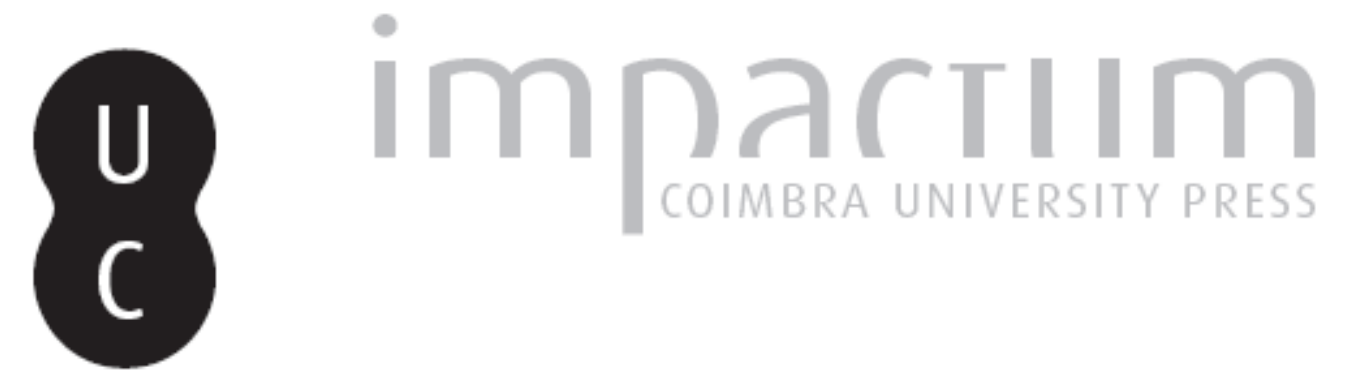

\title{
Aetates mortuorum: études quantitatives sur les âges des défunts en Lusitanie Autor(es): $\quad$ Curchin, Leonard A.
}

Publicado por: Imprensa da Universidade de Coimbra

URL persistente:

URI:http://hdl.handle.net/10316.2/45378

DOI:

DOI:https://dx.doi.org/10.14195/1647-8657_39_9

Accessed : $\quad$ 26-Apr-2023 00:28:02

A navegação consulta e descarregamento dos títulos inseridos nas Bibliotecas Digitais UC Digitalis, UC Pombalina e UC Impactum, pressupõem a aceitação plena e sem reservas dos Termos e Condições de Uso destas Bibliotecas Digitais, disponíveis em https://digitalis.uc.pt/pt-pt/termos.

Conforme exposto nos referidos Termos e Condições de Uso, o descarregamento de títulos de acesso restrito requer uma licença válida de autorização devendo o utilizador aceder ao(s) documento(s) a partir de um endereço de IP da instituição detentora da supramencionada licença.

Ao utilizador é apenas permitido o descarregamento para uso pessoal, pelo que o emprego do(s) título(s) descarregado(s) para outro fim, designadamente comercial, carece de autorização do respetivo autor ou editor da obra.

Na medida em que todas as obras da UC Digitalis se encontram protegidas pelo Código do Direito de Autor e Direitos Conexos e demais legislação aplicável, toda a cópia, parcial ou total, deste documento, nos casos em que é legalmente admitida, deverá conter ou fazer-se acompanhar por este aviso.

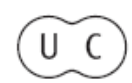


UNIVERSIDADE DE COIMBRA

FACULDADE DE LETRAS

\section{CONIMBRIGA}

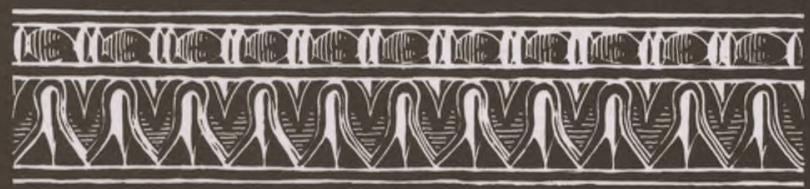

VOLUME XXXIX - 2000 
LEONARD A. CURCHIN

University of Waterloo (Canadá)

\section{AETATES MORTVORVM: ÉTUDES QUANTITATIVES SUR LES ÂGES DES DÉFUNTS EN LUSITANIE \\ "Conimbriga" XXXIX (2000) p. 275-284}

RESUMO: Uma análise quantitativa das inscrições funerárias da Lusitânia romana permite levantar duas questões acerca do significado da menção da idade dos defuntos.

Em primeiro lugar, considera-se a hipótese de R. Sailer e B. Shaw, segundo a qual os epitáfios de menores de dez anos seriam mais numerosos nas regiões em que se registasse uma forte representação de memórias postas pelos pais aos filhos. O Autor mostra que, na Lusitânia, apesar de tais monumentos serem proporcionalmente mais abundantes que em Roma, a proporção de epitáfios a crianças é muito modesta. Os testemunhos lusitanos refutam, pois, essa hipótese.

Analisa-se, depois, a extensão do analfabetismo na Lusitânia, na medida em que, na opinião do Autor, o arredondamento da idade seria disso um sintoma evidente. Ressalta, assim, que a proporção de idades arredondadas - e, por consequência, a extensão do analfabetismo - é bem maior na Lusitânia oriental que nos dois conventus atlânticos. Evidencia-se, além disso, o equívoco de R. Duncan-Jones, que, ao estudar o analfabetismo provincial, se não apercebeu de que cada região da Península Ibérica apresenta diferentes índices de arredondamento das idades.

RÉSUMÉ: Des analyses quantitatives de 1'épigraphie lusitanienne nous permettent d'aborder deux questions sur la signification des âges enregistrés sur les épitaphes romaines.

D'abord, on met à l'épreuve une hypothèse de R. Sailer et B. Shaw, que les épitaphes de personnes ágées de moins de dix ans seront plus nombreuses dans des régions avec une forte représentation de commémorations faites par des parents à ses enfants. L'auteur montre qu'en Lusi- 
tanie, bien que la proportion de commémorations descendantes soit plus haute qu'á Rome, la proportion d'épitaphes infantiles est trés modeste. Alors, l'évidence lusitanienne réfute cette hypothése.

Ensuite, l'auteur examine l'étendue de l'analphabétisme en Lusitanie, dans la mesure oü cela se révéle dans l'arrondissement des áges sur les épitaphes. II ressort que la proportion d'áges arrondis, et d'ici l'étendue de l'analphabétisme, est bien plus forte dans la Lusitanie orientale que dans les deux conventus atlantiques. On met en évidence aussi la méprise de R. Duncan-Jones, qui en étudiant l'analphabétisme provinciale n'a pas aperçu que chaqué région de la Péninsule Ibérique aurait un différent indice d'arrondissement des áges. 


\section{AETATES MORTVORVM: ÉTUDES QUANTITATIVES SUR LES ÂGES DES DÉFUNTS EN LUSITANIE}

Dans son ouvrage Péri makrobion ("Sur ceux qui ont eu la vie longue"), écrit à l'époque d'Hadrien, Phlegon de Trallès enregistre sept personnes de la Lusitanie qui ont atteint l'âge de cent ans: trois d'Interannia deux de Conimbriga, un d'“Apeilokarios" (Abelterium) et un d' "Aibourobisyngesia" (lEburobrittium) ${ }^{1}{ }^{2}$. Cet intérêt antique à la durée de la vie se réflète encore dans l'enregistrement de l'âge des défunts sur les épitaphes de la Lusitanie, qui nous apportent non seulement des exemples additionnels de centenaires (voir Table I), mais aussi des données utiles sur toute la série des âges. Bien que ce témoignage ne nous fournisse pas un vrai échantillon démographique - en raison de l'inégalité de la distribution géographique et chronologique des inscriptions, la pénurie d'épitaphes d'enfants, de pauvres et d'esclaves, et l'inexactitude des âges ${ }^{3}$ - il est néanmoins susceptible d'analyses qui peuvent éveiller l'intérêt des étudiants de l'histoire sociale. Dans cet article, en suivant les méthodes développées par d'autres savants, nous nous servirons des âges sur les inscriptions pour examiner deux aspects de la société lusitanienne: l'importance de la famille nucléaire, et la relation entre l'arrondissement des âges et l'analphabétisme.

1 Hellénisée par Phlegon en "Interamnesia", ceci doit être le chef-lieu des Interannienses (Pline, Nat. hist., 4.118; CIL, II, 760 (attestation comme municipiuni) $\mid H E p$., $1,210)$, dans la région de Viseu.

2 F. JАСОВ Y, Die Fragmente der griechischen Historiker, tome II, Leyde, 1962, p. 1187.

3 Voir C. Garcia Merino, Poblaciôn y poblamiento en Hispania romana: El conventus cluniensis, Valladolid, 1975, pp. 10-14. 
1. Les âges et la famille nucléaire

Il y a quelques années, R. Saller et B. Shaw ont modifié d'une manière importante le modèle traditionnel de la famille étendue à Rome, en montrant à partir des inscriptions funéraires que la plupart des commémorations traitent de la famille nucléaire (un homme, sa femme et leurs enfants). Parmi la population civile de Rome et de l'Italie, par exemple, entre $72 \%$ et $79 \%$ des commémorations appartiennent à ce type. Dans les provinces hispaniques, le chiffre est même plus haut, $83 \%{ }^{4}$. En dedans de la famille nucléaire, Saller et Shaw ont distingué des commémorations conjugales (entre des époux), descendantes (d'un parent ou des deux parents à son enfant), ascendantes (d'un enfant à son parent) et fraternelles (entre frères et soeurs). Encore selon les calculs de Saller et Shaw, les commémorations descendantes représentent $37 \%$ des commémorations nucléaires de sénateurs et de chevaliers, 33\% parmi les ordres inférieurs à Rome, et entre $22 \%$ et $43 \%$ dans les provinces (36\% en Hispanie). Des chiffres que nous avons computés pour la Lusitanie sont pratiquement les mêmes que Saller et Shaw ont constatés pour la Péninsule Ibérique dans son ensemble: 82\% des commémorations funéraires sont de type nucléaire, et parmi ces inscriptions nucléaires, $37 \%$ sont des commémorations descendantes ${ }^{5}$.

Un autre indice de l'importance de la famille nucléaire est fourni, selon Saller et Shaw, par les âges des défunts. Ces auteurs ont remarqué que dans des échantillons où il y a une haute proportion de commémorations descendantes, il y a une plus grande tendance à commémorer les enfants de moins de dix ans. Par exemple, parmi les basses classes à Rome, où les commémorations descendantes représentent $33 \%$ des exemples de la famille nucléaire, $39 \%$ des personnes dont l'âge est connu sont des enfants de moins de dix ans. Par contre, en Narbonnaise, où seulement $24 \%$ des commémorations nucléaires sont du type descendant, seulement $23 \%$ des âges préservés représentent de tels enfants ${ }^{6}$.

4 R. SALLER et B. SHaw, Tombstones and Roman family relations in the Principate: Civilians, soldiers and slaves, Journal of Roman Studies, 74, 1984, pp. 147-148.

5 L. A. Curchin, La famille lusitano-romaine, Actas de la IV Mesa Redonda de la Lusitania romana (Mérida, 2-4 de marzo de 2000), à paraître.

6 SALler et SHAw, art. cit., pp. 137-138. 
Alors, en Lusitanie, où les relations descendantes représentent $37 \%$ des commémorations nucléaires, on doit anticiper - si l'argument de Saller et Shaw est solide - que $40 \%$ ou plus des âges seront inférieurs à dix ans. Mais au contraire, de tels âges ne représentent que 9-10\% de l'échantillon lusitanien, dans n'importe quel conventus (Table I) ${ }^{7}$. Ce résultat inattendu paraît réfuter la théorie de Saller et Shaw; la tendance prétendue qu'ils ont aperçue à Rome et en Gaule méridionale doit donc être un effet de coïncidence. La situation en Lusitanie pourrait se comparer avec ce que l'on a trouvé dans les cimitières pré-romaines dans la plupart du monde celtique, c'est-à-dire que les jeunes enfants son peu représentés, ce qui pourrait suggérer que de tels enfants étaient vus en tant qu'une catégorie spéciale qui ne recevraient pas les mêmes rites funéraires que les adultes ${ }^{8}$.

Bien sûr, la distribution des âges dans les inscriptions ne nous offre pas de profil de la durée de la vie. L'on a estimé raisonnablement que dans le monde romain, la moitié des enfants sont morts sans achever les dix ans; alors, les indications d'âge dans l'épigraphie funéraire ne représente pas l'état réel de la mortalité antique ${ }^{9}$. En Lusitanie, évidemment, une grande partie des morts infantiles ne sont pas enregistrées sur les inscriptions, soit à cause des frais d'une pierre tombale, soit à cause d'une croyance qu'une épitaphe n'est pas nécessaire pour ce qui n'est pas arrivé à l'état adulte. Exceptionnellement, certes, il y avait des monuments plus chers - par exemple, la grande cupa en mar-

7 Pour des raisons que l'on a discutées ailleurs (J. Francisco Martin, Cuestiones en tomo al limite occidental de la Bética, Actas del 1 congreso de historia de Andaluci'a: Fuentes y metodologi'a, Andaluci'a en la Antigüedad, Cordoue, 1978, pp. 179-185; J. AlarCÂo et autres, Propositions pour un nouveau tracé des limites anciennes de la Lusitanie romaine, Les villes de Lusitanie romaine: Hiérarchies et territoires, Paris, 1990, pp. 325-327; J. D'ENCARNAÇÂo, Epigrafia romana de Moura, Moura na época romana, Moura, 1990, p. 41) j'ai inclus les concelhos de Serpa et Moura dans le conventus Pacensis, et les districts de Guarda et Castelo Branco dans le conventus Emeritensis. Toutefois il faut signaler que, faute de critères décisifs, les limites de conventus ne peuvent être qu'approximatifs.

8 T. Champion, Power, politics and status, The Celtic world, éd. M. J. Green, Londres et New-York, 1995, p. 90.

9 S.M. Treggiari, Roman marriage, Oxford, 1991, p. 398; B. BoY AVAL, Épigraphie antique et démographie: Problèmes de méthode, Revue du Nord, 59, 1977, pp. 163-191.

Conimbriga, 39 (2000) 275-284 
ble de Cogitata, âgée de cinq ans, qui provient probablement des environs de Myrtilis - mais la plupart des enfants morts en manquaient ${ }^{10}$.

En regardant seulement les commémorations de parents à ses enfants (Table II), nous voyons qu'au conventus Emeritensis 52\% des âges sont ceux d'enfants $(19 \%)$ ou de jeune adultes inférieurs à vingt ans $(33 \%)$. Par contre, $71 \%$ des défunts de Pacensis, et $63 \%$ de ceux de Scallabitanus, ont vingt ans ou plus. Ces résultats ne veulent pas dire que la durée de la vie était beaucoup plus courte au conventus de Mérida. Plutôt ils indiquent une plus forte tendance dans l'Emeritensis à commémorer des petits enfants et des adolescents. D'ailleurs, dans tous les conventus il y a un grand nombre de jeunes adultes de moins de trente ans. Des historiens de la famille ont déjà remarqué que, du point de vue des parents, la mort de ces jeunes gens était particulièrement triste, parce qu'en survivant l'enfance ils avaient donné aux parents un faux espoir qu'ils vivraient longtemps ${ }^{n}$. Alors il y avait une tendance prononcée à leur faire une commémoration.

\section{Les âges et l'analphabétisme}

Dans une étude célèbre, R.P Duncan-Jones a montré qu'il se passe souvent que des personnes analphabètes ignorent leur âge exact. L'analphabétisme se révèle donc quand un nombre disproportionné d'âges enregistrés sur des épitaphes sont divisibles par cinq, ce qui indique que de tels âges sont des approximations ${ }^{12}$. D'ailleurs, Duncan-Jones a constaté que des âges arrondis se trouvent dans l'épigraphie romaine de la Péninsule Ibérique plus fréquemment qu'en Italie ou en Gaule, mais moins fréquemment que dans les provinces rhéniennes et danubiennes ${ }^{13}$.

10 J. D'EnCarnação, Inscrições romanas do Conventus Pacensis, Coimbra, $1984,{ }^{\circ}{ }^{\circ} 105$.

11 S. Dixon, The Roman family, Londres et Sydney, 1988, p. 100; S. L. Dyson, Community and society in Roman Italy, Baltimore et Londres, 1992, p. 182.

12 L'approximation des âges peut se révéler aussi par la phrase plus minus, qui se trouve parfois en Lusitanie: ENCARNAÇão, Inscrições, n. ${ }^{\circ} 23$ et 134; ILER, n. ${ }^{\circ} 3678$. Même quand une épitaphe, avec un âge divisible par cinq, mentionne le nombre de mois et de jours que le défunt a vécu, ceci ne prouve pas que le nombre d'ans est exact. On pourrait savoir la date de son anniversaire, sans savoir l'an de sa naissance.

13 R.P. DUNCAN-Jones, Age-rounding, illiteracy, and social différentiation in the Roman Empire, Chiron, 7, 1977, pp. 333-353.

Conimbriga, 39 (2000) 275-284 
L'étude de Duncan-Jones est très importante, mais ses résultats pour les provinces hispaniques sont quelque peu trompeurs, parce qu'il tient la Péninsule pour une région homogène. Au contraire, le taux d'analphabétisme doit être moins fort dans les zones bien romanisées, par exemple la Bétique et la Catalogne, et plus fort dans les zones écartées. Ainsi que nous avons indiqué dans un article précédent, la tendance à arrondir les âges est beaucoup moins marquée à Tarraco, et beaucoup plus marquée en Celtibérie, que dans la Péninsule en moyenne ${ }^{14}$. Alors, on peut s'attendre qu'en Lusitanie aussi, l'étendue de l'arrondissement des âges, et par conséquent de l'analphabétisme, différera de celle que Duncan-Jones a calculée pour l'Hispanie généralement. Mais est-ce que le degré d'arrondissement d'âges en Lusitanie sera plus grand ou plus petit que la moyenne péninsulaire?

Pour résoudre cette question, nous avons suivi la formule utilisée par Duncan-Jones, en excluant les âges d'enfants, d'adolescents et de personnes âgées. Pour chaque décade entre les âges 23-62 il faut calculer le pourcentage d'âges divisibles par cinq. Ensuite, on prend la moyenne de ces quatres pourcentages. Pour trouver la déviation d'une distribution normale (où $20 \%$ des âges seraient divisibles par cinq), il faut soustraire 20 de cette moyenne et multiplier par 1,25. Ces calculs nous donnent un indice d'arrondissement des âges, sur une échelle de $0-100$.

Pour la Péninsule en totalité, Duncan-Jones a calculé un indice d'arrondissement de $56,6 \%$ pour les hommes et $58,4 \%$ pour les femmes. (L'indice féminin est normalement plus haut que le masculin, parce qu'en général les garçons recevaient une meilleure formation.) En Lusitanie, par contre, les indices sont sensiblement plus hauts: $64,3 \%$ pour hommes, $66,1 \%$ pour femmes (Table III). Ces chiffres suggèrent fortement un taux d'analphabétisme assez haut en Lusitanie (mais plus bas qu'en Celtibérie, où les indices correspondants sont de $73,5 \%$ et $74,6 \%$ ). Même dedans la Lusitanie, on trouve entre les trois conventus des indices différents, qui nous apportent une surprise. On aurait s'attendre à un taux d'arrondissement plus bas dans le conventus Emeritensis, où se trouva la capitale provinciale. Au contraire, les indices pour Emeritensis sont de $69,4 \%$ et $71,4 \%$, les plus hauts de la province, tandis que Pacensis et Scallabitanus ont des indices assez

14 L. A. CURCHIN, Literacy in the Roman provinces: Qualitative and quantitative data from central Spain, American Journal of Philology, 116, 1995, pp. 471-472. 
proches des moyennes hispaniques calculées par Duncan-Jones. Ces chiffres suggèrent que l'analphabétisme était plus prononcé dans $\mathrm{L}$ arrière-pays de la province que dans les zones littorales.

En regardant les indices urbains et ruraux (Table III), on voit que l'arrondissement des âges était plus commun dans les campagnes que dans les villes, surtout pour les hommes (une différence de presque dix pour-cent entre hommes urbains et ruraux), ce qui s'expliquerait par un plus haut degré de romanisation et d'éducation dans le secteur urbain. Dans la campagne, par contraste, l'indice masculin est tellement haut, qu'il surpasse même l'indice féminin. On trouve aussi un indice féminin très bas dans Pacensis, qui pourrait cependant s'attribuer à la petitesse de l'échantillon.

\section{Conclusions}

Ces deux études quantitatives des âges en Lusitanie romaine ont eu des résultats significatifs. D'une part, les données pour la Lusitanie réfutent par exemple l'hypothèse de Saller et Shaw, que les épitaphes d'enfants de moins de dix ans sont plus fréquentes dans les régions avec une prépondérance de commémorations descendantes. Néanmoins, la forte proportion de telles commémorations confirme l'importance de la famille nucléaire en Lusitanie.

D'autre part, les indices d'arrondissement des âges, et par conséquent le taux d'analphabétisme, dans le conventus de Mérida sont beaucoup plus hauts que les indices pour l'Hispanie publiés par Duncan-Jones. Ces résultats paraissent réfléchir la romanisation incomplète des Vettons. Par contre, le taux d'arrondissement est assez modeste dans les deux conventus côtiers. 
TABLE I - Commémorations funéraires: âge du défunt

\begin{tabular}{|c|rr|rr|rr|}
\hline & \multicolumn{2}{|c|}{ Pacensis } & \multicolumn{2}{|c|}{ Scallabitanus } & \multicolumn{2}{|c|}{ Emeritensis } \\
\hline ans & $\mathrm{N}$ & $\%$ & $\mathrm{~N}$ & $\%$ & $\mathrm{~N}$ & $\%$ \\
$0-9$ & 26 & 9 & 22 & 9 & 98 & 10 \\
$10-19$ & 36 & 13 & 46 & 19 & 117 & 12 \\
$20-29$ & 55 & 20 & 66 & 27 & 179 & 19 \\
$30-39$ & 56 & 20 & 42 & 17 & 128 & 13 \\
$40-49$ & 24 & 9 & 23 & 9 & 86 & 9 \\
$50-59$ & 31 & 11 & 16 & 6 & 136 & 14 \\
$60-69$ & 22 & 8 & 19 & 8 & 103 & 11 \\
$70-79$ & 12 & 4 & 10 & 4 & 63 & 7 \\
$80-89$ & 12 & 4 & 2 & 1 & 36 & 4 \\
$90-99$ & 2 & 1 & 0 & 0 & 5 & 1 \\
$100-109$ & 0 & 0 & 2 & 1 & 6 & 1 \\
& 276 & 100 & 248 & 100 & 957 & 100 \\
\hline
\end{tabular}

TABLE II - Commémorations descendantes: âge du défunt

\begin{tabular}{|c|c|c|c|c|c|c|}
\hline \multirow[b]{2}{*}{ ans } & \multicolumn{2}{|c|}{ Pacensis } & \multicolumn{2}{|c|}{ Scallabitanus } & \multicolumn{2}{|c|}{ Emeritensis } \\
\hline & $\mathrm{N}$ & $\%$ & $\mathrm{~N}$ & $\%$ & $\mathrm{~N}$ & $\%$ \\
\hline $0-9$ & 4 & 11 & 12 & 13 & 24 & 19 \\
\hline $10-19$ & 7 & 19 & 22 & 24 & 41 & 33 \\
\hline $20-29$ & 18 & 49 & 35 & 38 & 39 & 31 \\
\hline $30-39$ & 8 & 22 & 18 & 19 & 17 & 14 \\
\hline $40-49$ & 0 & 0 & 5 & 5 & 3 & 2 \\
\hline \multirow[t]{2}{*}{$50-59$} & 0 & 0 & 1 & 1 & 1 & 1 \\
\hline & 37 & 100 & 93 & 100 & 125 & 100 \\
\hline \multirow{2}{*}{$\begin{array}{l}0-19 \text { total } \\
20-59 \text { total }\end{array}$} & 11 & 30 & 34 & 37 & 65 & 52 \\
\hline & 26 & 71 & 59 & 63 & 60 & 48 \\
\hline
\end{tabular}

Conimbriga, 39 (2000) 275-284 
TABLE III - Arrondissement des âges en Lusitanie

(Le nombre d'examples dans $V$ ¿chantillón s'indique entre parenthèses)

\begin{tabular}{|l|c|c|}
\hline & $\begin{array}{c}\text { índice d'arrondissement: } \\
\text { HOMMES }\end{array}$ & $\begin{array}{c}\text { índice d'arrondissement: } \\
\text { FEMMES }\end{array}$ \\
\hline Pacensis & $56,8(75)$ & $53,3(69)$ \\
Scallabitanus & $55,1(73)$ & $60,9(60)$ \\
Emeritensis & $69,4(268)$ & $71,4(225)$ \\
domaine urbain & $59,6(214)$ & $66,1(182)$ \\
domaine rural & $69,1(202)$ & $67,3(172)$ \\
toute Lusitanie & $64,3(416)$ & $66,1(354)$ \\
\hline
\end{tabular}

